\title{
comportement tridimensionnel des sables: comparaison d'essais véritablement triaxiaux et d'essais sur cylindre creux
}

\author{
comparison of three dimensional tests performed \\ with a true triaxial apparatus \\ and a hollow cylinder device
}

\author{
J. LANIER, Z. ZITOUNI \\ Institut de Mécanique de Grenoble \\ A. SAADA, P. PUCCINI, G. BIANCHINI \\ Case Western Reserve University* *
}

Rev. Franç. Géotech. $\mathrm{n}^{\circ} 49$, pp. $67-76$ (octobre 1989)

\section{Résumé}

Un programme de recherche mené en parallèle entre l'Institut de Mécanique de Grenoble et l'Université de Cleveland a permis d'étudier le comportement de deux sables fins à l'aide de deux appareils différents: une presse 3D à face rigide et un appareil de compression-torsion sur cylindre creux. Nous présentons dans cet article les résultats obtenus sur chemin isotrope et sur une série de chemins radiaux $(b=c t e)$. La méthode de comparaison mise en œuvre consiste, à partir des résultats expérimentaux obtenus sur la presse $3 \mathrm{D}$, à calculer les grandeurs mesurées au cylindre creux. La comparaison des essais isotropes se révèle délicate et les mesures ne deviennent semblables qu'après des corrections importantes dues à la pénétration de membrane; par contre on observe une bonne cohérence entre les essais radiaux réalisés sur les deux appareils.

\footnotetext{
Abstract

A joined research between Institut de Mécanique de Grenoble and Cleveland University was concerned with the behavior of two fine sands. Two different apparatuses were used for this study: a true triaxial apparatus and a hollow cylinder device. A comparison of the obtained data is achieved by the following method: from experimental results on the true triaxial apparatus, the quantities measured on the hollow cylinder are calculated and then compared to real data. For results concerned with isotropic tests, some discrepancy is observed which can be reduced by large corrections with respect to membrane penetration. But, for a series of radial tests $(\mathrm{b}=$ constant), data from the two apparatuses are in a rather good agreement.
}

\footnotetext{
- Domaine universitaire, BP 53 X, 38041 Grenoble Cedex

Cleveland, Ohio, USA.
} 


\section{INTRODUCTION}

L'étude expérimentale du comportement tridimensionnel des géomatériaux a connu un développement important au cours des quinze dernières années. Pendant longtemps les seuls essais réalisés en laboratoire étaient les essais triaxiaux classiques ou les essais à la boite de cisaillement. Depuis les années 70 des appareillages plus sophistiqués ont été développés: les presses tridimensionnelles qui permettent de faire varier indépendamment les trois contraintes principales agissant sur un échantillon parallélépipédique, les presses de compression-torsion sur cylindre creux, les cellules de cisaillement direct (DSC). Pour une description détaillée de ces appareils on pourra consulter les références $1,2,3$.

Parallèlement, de nombreuses lois de comportement ont été proposées dans la littérature; leurs possibilités et limitations ont été testées à loccasion de plusieurs Workshops $(4,5,6)$. La démarche utilisée est maintenant une procédure classique: les paramètres du modèle sont calés à partir d'essais classiques (triaxiaux de révolution, essais isotropes) et sa validation est obtenue ensuite par comparaision entre ses prédictions numériques et les résultats expérimentaux d'essais plus complexes, généralement tridimensionnels. C'est donc tout naturellement l'expérience qui, en dernier lieu, permet d'apprécier la qualité des modèles proposés. En cas de divergence, on voit souvent s'élever des critiques sur la qualité des essais, soulignant certaines «incohérences » expérimentales.

Notre but n'est pas de prétendre que les essais sont parfaits. Même pour des matériaux de laboratoire pour lesquels la granulométrie est bien définie, la difficulté de reproduire des essais est bien connue. Les causes en sont diverses: la préparation des échantillons, la mise en place sur les machines d'essais, l'homogénéité initiale et en cours d'essais, les phénomènes de localisation de déformation, sans parler des difficultés de métrologie.

La comparaison d'essais triaxiaux classiques effectués par différents laboratoires (7), dans des conditions théoriquement identiques, avec le même matériau, est de ce point de vue éloquente: si l'on peut considérer que le même essai répété dans un même laboratoire, avec le même expérimentateur et la même presse, est dans une large mesure reproductible, la comparaison des résultats interlaboratoires montre une dispersion qui peut être importante.

Notre démarche dans cet article est similaire et, si l'on peut dire, plus ambitieuse, puisqu'il s'agit de comparer des résultats expérimentaux théoriquement «semblables* obtenus dans deux laboratoires sur deux appareils de principe différent. En effet à l'occasion d'une coopération entre l'Institut de Mécanique de Grenoble (IMG) et Case Western Reserve University (CWRU) de Cleveland, une large campagne d'essais a été menée, sur une presse tridimensionnelle (IMG) et une presse de traction-torsion sur cylindre creux (CWRU). Nos résultats ont servi d'essais de référence pour un workshop sur les lois de comportement (6).
Ils sont actuellement disponibles dans une banque de données internationale à Cleveland.

Nous proposons ici une analyse comparative des résultats obtenus. Nous décrivons d'abord les deux appareils utilisés et le programme expérimental réalisé, puis nous comparons les résultats obtenus, en particulier sur le sable d'Hostun qui a récemment été choisi comme matériau test du $\approx$ Benchmarks for Geotechnical Modelling» (Banque de données pour la modélisation. créée à l'initiative de O.C. ZIENKIEWICZ et M. PASTOR en cours d'installation à l'Ecole Centrale de Paris (8)). Nous donnerons enfin quelques conclusions concernant la «précision » que l'on peut attendre des essais et l'anisotropie initiale des échantillons.

\section{LA PRESSE TRIDIMENSIONNELLE DE GRENOBLE}

Cet appareil conçu et réalisé à l'IMG est particulièrement adapté à l'étude du comportement en grandes déformations des milieux pulvérulents $(9,10,11)$. L'échantillon parallélépipédique est enfermé entre six faces rigides (fig. 1). Ses dimensions peuvent varier de 5 à $15 \mathrm{~cm}$ avec une vitesse maximale de $5 \mathrm{~mm} / \mathrm{min}$. Six moteurs électriques, synchronisés 2 à 2 , permettent le contrôle cinématique de l'essai.

L'échantillon, enveloppé dans une membrane en latex, de dimensions $100 \times 100 \times 100 \mathrm{~mm}^{3}$ et d'épaisseur $0,3 \mathrm{~mm}$, est préparé en dehors de la machine dans un moule. Le matériau est soit damé soit vibré pour avoir des échantillons denses, ou déposé avec une hauteur de chute nulle pour avoir des échantillons lâches. Il est ensuite graissé et transporté vers la machine avec un vide intérieur pour éviter le remaniement. La graisse sert à éliminer les contraintes tangentielles parasites qui peuvent se développer à l'interface plaques-échantillon.

En cours d'essai, trois capteurs de pression, noyés dans les plaques et insensibles aux contraintes tangentielles, mesurent directement les trois contraintes nor-

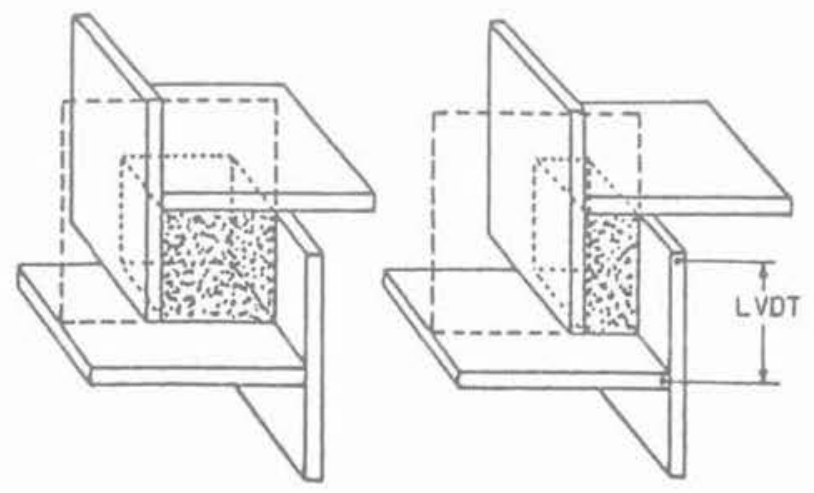

Fig. 1. - Principe de fonctionnement de la presse 3D de Grenoble.

Fig. 1. - Principle of the Grenoble true triaxial apparatus with rigid boundaries 
males aux faces que l'on considère (en négligeant le frottement) comme les trois contraintes principales $\sigma_{i}$.

Trois autres capteurs de type LVDT mesurent les dimensions de l'échantillon. Les déformations principales $\epsilon_{\mathrm{i}}$ sont calculées par:

$$
\epsilon_{\mathrm{i}}=-\log \left(\mathrm{X}_{\mathrm{i}} / \mathrm{X}_{\mathrm{io}}\right)
$$

Les variations de volume sont obtenues par:

$$
\epsilon_{\mathrm{v}}=-\log \left(\mathrm{V} / \mathrm{V}_{0}\right)
$$

A chaque mesure, on connaît donc: les trois valeurs principales de l'état de contrainte $\sigma_{1}$ et les trois valeurs principales de l'état de déformation $\epsilon_{\mathrm{j}}$.

Les difficultés rencontrées sont essentiellement de deux types:

1. Difficultés de préparation d'échantillons rigoureusement identiques, et difficultés de mise en place de l'échantillon sur la presse.

2. Apparition de la localisation de la déformation dans des bandes de cisaillement. Il y a alors perte de l'homogénéité et les mesures globales que nous enregistrons ne sont plus significatives.

Signalons enfin que les régulations sur chaque direction sont indépendantes et peuvent être soit cinématiques (contrôle de la vitesse des moteurs) soit en contrainte par un asservissement de type analogique.

\section{CYLINDRE CREUX DE CLEVELAND}

\subsection{Présentation de l'appareil}

La presse de compression-torsion de Cleveland a été un des premiers appareils de ce type utilisé pour l'étude du comportement des sols.

L'échantillon a la forme d'un cylindre creux de dimensions: $H=13 \mathrm{~cm}, \Phi_{\mathrm{e}}=7.1 \mathrm{~cm}$ et $\Phi_{1}=5.1 \mathrm{~cm}$. Il est préparé entre deux membranes cylindriques à laide d'un moule. Le mode de densification est identique à celui utilisé à Grenoble (damage ou vibration). La base de lappareil est fixe et la tête (fig. 2) permet d'appliquer un effort axial (extension ou compression), et/ou un couple de torsion; l'asservissement de ces deux sollicitations est pneumatique. Un système de drainage permet la mesure des pressions interstitielles (essais non drainés) ou des variations de volume (essais drainés).

L'ensemble est placé dans une cellule triaxiale classique qui assure une pression de confinement identique à l'intérieur et à l'extérieur du cylindre.

En cours d'essai, les quantités mesurées sont les suivantes: la déformation axiale, la rotation de tête, la variation de volume (essai drainé), la pression de confinement, l'effort axial et le couple de torsion.

\subsection{Etat de contrainte}

Dans le cylindre creux, on suppose que l'état de contrainte ne dépend ni de $\theta$, ni de z (en excluant les effets de frettage en tête et pied). Il peut par contre être fonction de $r$. On peut déterminer, en fonction des conditions aux limites, des valeurs moyennes qui seront d'autant plus représentatives que le rapport $e / R$ sera faible $\left(e=R_{e}-R_{i}\right)$.

Si l'on note $\mathrm{p}$ la pression de confinement, $\mathrm{F}$ l'excédent de force axiale et $M_{1}$ le couple de torsion, on obtient:

$$
\begin{aligned}
& \sigma_{z}=\mathrm{p}+\mathrm{F} / \mathrm{S}=\mathrm{p}+\sigma \\
& \sigma_{\theta}=\sigma_{\mathrm{r}}=\mathrm{p} \\
& \tau_{\theta 2}=\tau=3 \mathrm{M}_{\mathrm{t}} / 2 \pi\left(\mathrm{R}_{\mathrm{e}}^{3}-\mathrm{R}_{\mathrm{i}}^{3}\right)
\end{aligned}
$$

La direction $r$ est une direction principale; les deux autres appartiennent au plan tangent du cylindre et font avec la direction $\mathrm{Z}$ un angle $\alpha$ tel que:

$$
\operatorname{tg} 2 \alpha=2 \tau / \sigma \sim M_{4} / F
$$

Les valeurs principales de l'état de contrainte peuvent être définies par:

$$
\begin{aligned}
& \sigma_{1}=\mathrm{p}+\tau / \operatorname{tg} \alpha \\
& \sigma_{2}=\mathrm{p} \\
& \sigma_{3}=\mathrm{p}-\tau \cdot \operatorname{tg} \alpha
\end{aligned}
$$

On en déduit une expression du coefficient b utilisé classiquement en mécanique des sols:

$$
\mathrm{b}=\frac{\sigma_{2}-\sigma_{3}}{\sigma_{1}-\sigma_{3}}=\sin ^{2} \alpha
$$

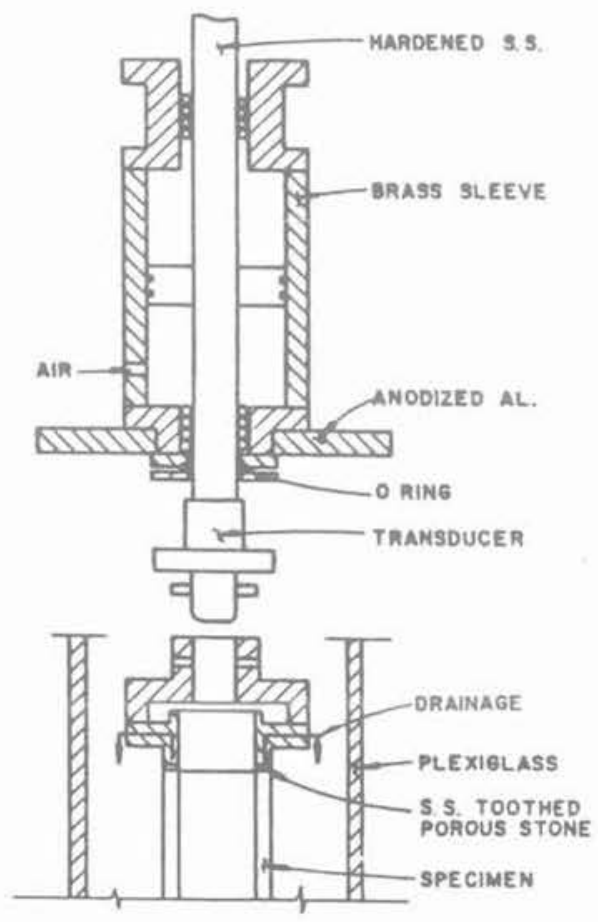

Fig. 2. - Principe du cylindre creux [15]. Fig. 2. - Schematic of hollow cylinder cell. 
Cette relation indique que les deux paramètres $b$ et $\alpha$ sont liés. On ne peut donc pas étudier séparément leur influence sur le comportement du matériau testé.

\subsection{Etat de déformation}

Dans l'hypothèse des petites déformations, la cinématique du cylindre creux est décrite par un champ de déplacement de la forme:

$$
\overrightarrow{\mathrm{u}}=\mathrm{u}_{\mathrm{r}} \vec{e}_{\mathrm{r}}+\mathrm{u}_{\theta} \vec{e}_{\theta}+\mathrm{u}_{\mathrm{z}} \vec{e}_{\mathrm{z}}
$$

avec: $u_{\mathrm{r}}=\mathrm{u}(\mathrm{r}) ; \mathrm{u}_{\theta}=\mathrm{krz} ; \mathrm{u}_{\mathrm{z}} ;=\lambda z$

On en déduit le tenseur de déformation $\epsilon$ et le vecteur rotation $\Omega$ par:

$$
\begin{aligned}
& \epsilon_{\mathrm{r}}=\mathrm{du} / \mathrm{dr} ; \epsilon_{\theta}=\mathrm{u} / \mathrm{r} ; \epsilon_{\mathrm{z}}=\lambda ; \gamma=\mathrm{kr} \\
& \vec{\Omega}=-(\mathrm{kr} / 2) \vec{e}_{\mathrm{r}}+\mathrm{kz} \overrightarrow{\mathrm{e}}_{\mathrm{z}}
\end{aligned}
$$

La connaissance complète de l'état de déformation nécessite donc quatre mesures pour la détermination de $\epsilon_{r}, \epsilon_{\theta}, \epsilon_{z}$ et $\gamma$.

S'il est relativement aisé de mesurer $\epsilon_{\mathrm{z}}$ (allongement axial) et $\gamma$, la détermination directe de $\epsilon_{\mathrm{r}}$ et $\epsilon_{\theta}$ est plus difficile car elle nécessite a priori les mesures de variations des rayons intérieur et extérieur. L'utilisation des jauges de proximité (12) s'est révélée peu fiable pour la géométrie des éprouvettes qui ont été utilisées.

Notons que la détermination des variations de volume $\epsilon_{\mathrm{v}}$ permet d'obtenir la somme $\epsilon_{\mathrm{r}}+\epsilon_{\theta}=\epsilon_{\mathrm{v}}-\epsilon_{\mathrm{z}}$.

KARCHAFI $(13,14)$ qui utilise un cylindre creux de dimensions plus importantes signale également la difficulté d'obtenir des mesures de $\epsilon_{\mathrm{r}}, \epsilon_{\theta}, \epsilon_{\mathrm{z}}$ et $\gamma$ cohérentes entre elles; le principal problème étant la détermination de $\epsilon_{\mathrm{r}-}$.

Pour comprendre d'où vient cette difficulté, on peut estimer une valeur moyenne de $\epsilon_{\mathrm{r}}$ de la façon suivante:

L'équation de compatibilité s'écrit:

$$
\mathrm{d}\left(\mathrm{r} \epsilon_{\theta}\right)=\epsilon_{\mathrm{r}} \mathrm{dr}
$$

En intégrant de $R_{i}$ à $R_{e}$ et en posant $R_{\text {moy }}=\left(R_{e}+\right.$ $\left.R_{i}\right) / 2$, e $=R_{e}-R_{i}$, on obtient:

$$
\begin{aligned}
& \left(\epsilon_{\mathrm{r}}\right)_{\text {moy }}=\frac{1}{e} \int_{\mathrm{R}_{\mathrm{i}}}^{\mathrm{R}_{e}} \epsilon_{\mathrm{r}} \mathrm{dr}= \\
& \frac{\epsilon_{\theta}\left(\mathrm{R}_{\mathrm{e}}\right)+\epsilon_{\theta}\left(\mathrm{R}_{\mathrm{i}}\right)}{2}+\frac{\mathrm{R}_{\text {moy }}}{e}\left(\epsilon_{\theta}\left(\mathrm{R}_{\mathrm{e}}\right)-\epsilon_{\theta}\left(\mathrm{R}_{\mathrm{j}}\right)\right)
\end{aligned}
$$

où l'on remarque que la différence $\left(\epsilon_{\theta}\left(\mathrm{R}_{e}\right)-\epsilon_{\theta}\left(\mathrm{R}_{\mathrm{j}}\right)\right)$ est multipliée par le coefficient amplificateur $\mathrm{R}_{\text {moy }} / \mathrm{e}$.

Nous avons donc là une difficulté inhérente à l'appareil de traction-torsion sur cylindre creux: une bonne approximation de l'état de contrainte est obtenue pour $e / R_{\text {moy }}$ petit et, dans ce cas, la détermination de $\left(\epsilon_{\mathrm{r}}\right)_{\text {moy }}$ devient incertaine.

Avant de conclure ce paragraphe sur l'étude de la déformation, nous voulons évaluer la rotation des directions matérielles dans le plan tangent du cylindre. Soit une direction $\mathrm{n}$ dans ce plan faisant l'angle $\alpha_{1}$ avec l'axe $\theta$. La rotation de cette direction par rapport aux axes $(\theta, z)$ est définie par:

$$
\delta \alpha_{1}=\sin \alpha_{1} .\left(\left(\epsilon_{z}-\epsilon_{\theta}\right) \cos \alpha_{1}-\gamma \sin \alpha_{1}\right)
$$

d'où l'on déduit que les directions principales de $\epsilon$ définies par:

$$
\left.\operatorname{tg} 2 \alpha=\gamma / \epsilon_{\theta}-\epsilon_{z}\right)
$$

subissent une rotation $\delta \alpha=-\gamma / 2$. Nous utiliserons cette remarque dans le paragraphe 4.2.1.

\section{COMPARAISONS DES RÉSULTATS D'ESSAIS}

Nous nous proposons dans ce paragraphe de comparer les résultats d'essais obtenus sur la presse 3D de l'IMG et sur le cylindre creux de Cleveland.

Les essais réalisés sur ces deux appareils ne sont pas tous comparables: en effet, sur les presses tridimensionnelles, les axes principaux de contrainte (et de déformation) restent fixes et confondus avec les directions matérielles de la machine; alors qu'au cylindre creux, les directions principales dans le plan tangent du cylindre définies par:

$$
\operatorname{tg} 2 \alpha=2 \pi / \sigma
$$

peuvent tourner en fonction de l'asservissement du rapport $\tau / \sigma$.

On ne peut donc songer à comparer que des essais tels que $\alpha=$ cte (essais sans rotation des directions principales) et en conséquence $b=$ cte (cf. § 3.2).

\subsection{Comparaison d'essais isotropes}

Les essais de compression isotrope réalisés sur les échantillons parallélépipédiques de la presse 3D et les cylindres creux doivent théoriquement, si les conditions de préparation sont identiques, donner le même résultat. On peut évoquer ici les effets de membrane; en effet, les déformations volumiques sont faibles et les variations importantes de la pression de confinement entraîne une surestimation (due à la membrane) de la diminution de volume quand $\mathrm{p}$ augmente. Selon (15), les corrections de ces effets sont peu fiables. La figure 3 donne un exemple de correction de pénétration de membrane pour un essai au cylindre creux. La réponse du matériau est alors proche de l'isotropie. Pour les essais sur presse 3D, les conditions aux limites avec faces rigides nécessiteraient des corrections de type "effet de paroi » qui sont faibles pour un sable fin. Ces corrections n'ont pas été faites. 

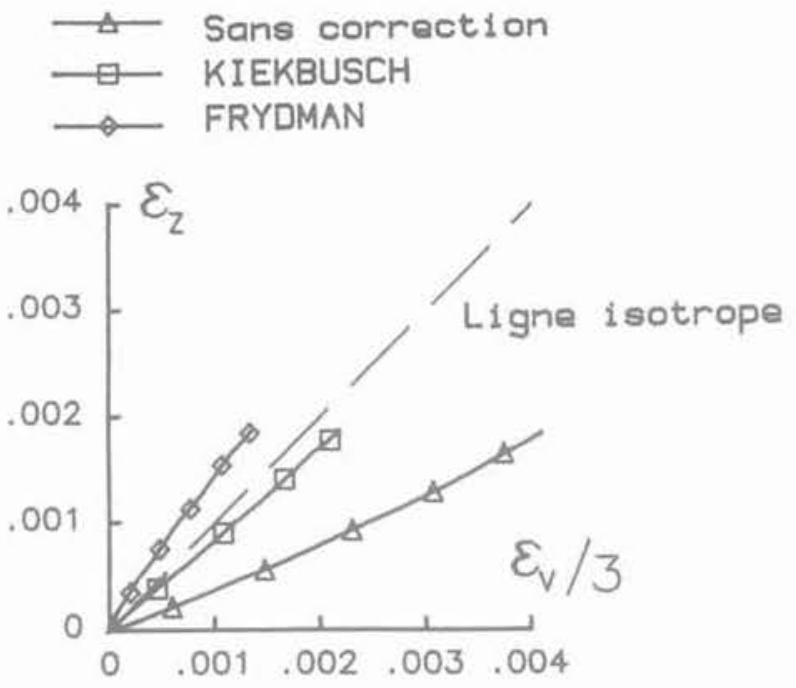

Fig. 3. - Correction de pénétration de membrane (Essai isotrope) [15]

Fig. 3, - Evaluation of membrane corrections under isotropic stress.

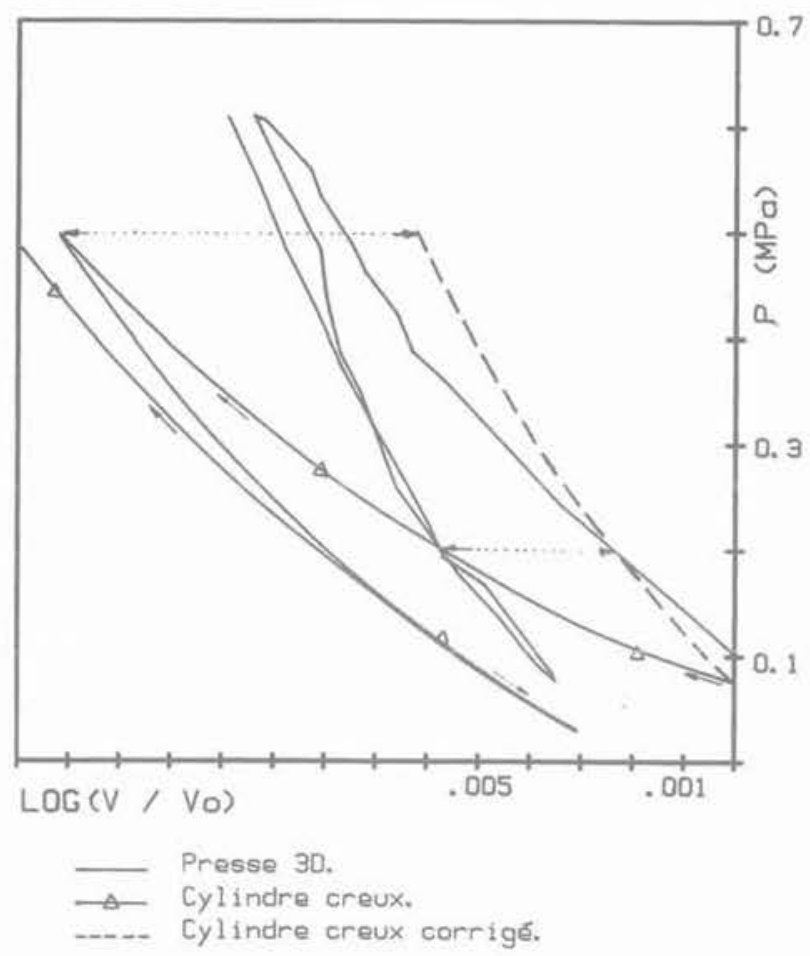

Fig. 4. - Comparaison des essais isotropes,

Fig. 4. - Comparison of data from isotropic tests.

La figure 4 donne les résultats bruts de deux essais isotropes, l'un réalisé au cylindre creux, l'autre sur la presse $3 \mathrm{D}$. On constate que pour une pression donnée, le serrage mesuré est plus important au cylindre creux. Notons cependant que si l'on utilise les corrections proposées sur la figure 3 (en supposant $\epsilon_{\mathrm{v}}$ $=3 . \epsilon_{z}$ ) les résultats deviennent tout à fait comparables.
$\mathrm{Ce}$ n'est donc que grâce à des corrections incertaines que nos résultats deviennent concordants. Il est donc délicat d'en tirer des conséquences de nature rhéologique. Ce point est important à signaler car beaucoup de modèles utilisent ce type d'essai pour le calage de leurs paramètres.

\subsection{Comparaison d'essais à $\mathrm{b}=\mathrm{cte}$}

\subsubsection{Définition des essais}

Les essais réalisés au cours de la collaboration de nos deux laboratoires nous ont permis d'étudier le comportement de plusieurs sables, en particulier sur les chemins définis par: $\sigma_{2}=$ cte et $\mathrm{b}=$ cte. Il s'agit donc de chemin ayant la même histoire de valeurs principales.

A la presse 3D, ces essais sont obtenus en régulant: - une vitesse d'écrasement sur la direction 1 d'où il résulte une valeur $\sigma_{1}$;

$-\sigma_{2}=$ cte;

$-\sigma_{3}=\left(\sigma_{1}-\right.$ b. $\left.\sigma_{2}\right) /(1-$ b $)$;

où la direction principale majeure 1 est une direction matérielle (axe vertical de la machine).

Au cylindre creux, la condition $\sigma_{2}=$ cte impose une pression constante de cellule (il n'y a donc pas à considérer de correction due à la pénétration de membrane). La condition $\mathrm{b}=$ cte est équivalente à $\alpha=$ cte (cf. § 3.2). Il est donc nécessaire de réaliser un asservissement entre le couple de torsion et l'effort axial tel que: $M_{1} / F=$ cte. Les directions principales de l'état de contrainte sont alors fixes par rapport au laboratoire mais ne correspondent pas, contrairement aux essais sur presse 3D, à des directions matérielles. Leur rotation dans le plan tangent du cylindre peut être évaluée par $\delta \alpha$ (cf. § 3.3). Des mesures effectuées par KARCHAFI (13) et SAADA (16), montrent que les directions principales de contrainte et de déformation restent voisines et que l'écart maximum, pour différentes valeurs de b est d'environ $5^{\circ}$. On peut donc estimer la rotation des directions matérielles coïncidant avec les directions principales de contrainte par $\delta \alpha=-\gamma / 2$, soit $2^{\circ}, 5$ pour $\gamma=16 \%$. Cette rotation est faible et par conséquent justifie la comparaison des essais à $b=$ cte au cylindre creux et sur presse 3D. Une étude plus complète, en grandes déformations, de la comparaison des essais sur les deux appareils est publiée en (17).

\subsubsection{Méthode de comparaison}

Les mesures effectuées sur chaque appareil ne correspondent pas aux mêmes systèmes d'axes: $(r, \theta$, z) pour le cylindre creux, (1, 2, 3 = axes principaux) pour la presse 3D. Il est donc nécessaire de se ramener à des quantités équivalentes pour les deux types d'essais. Une première méthode consisterait à comparer l'évolution des valeurs principales (ou des invariants), mais nous avons vu qu'il était difficile d'estimer $\epsilon_{\mathrm{r}}=\epsilon_{2}$ au cylindre creux. Nous proposons donc, à partir des valeurs principales mesurées sur presse $3 \mathrm{D}$, de "revenir» aux mesures effectuées aux cylindres creux en calculant le vecteur contrainte 
$\left(\sigma_{z}{ }^{*}, \tau_{z}{ }^{*}\right)$ d'une part, et $\left(\epsilon_{z}{ }^{*}, \gamma^{*}\right)$ correspondant à une direction $z^{*}$ de l'éprouvette parallélépipédique, telle que $\left(\mathrm{z}^{*}, 1\right)=\alpha$, où $\alpha$ est défini par $\mathrm{b}=\sin ^{2}$ $\alpha$ (fig. 5). On pourra alors faire une comparaison directe des valeurs ainsi calculées avec les mesures réalisées au cyclindre creux dans l'hypothèse où le matériau est isotrope.

Notons à ce propos que, les participants au Workshop de Cleveland devaient simuler les essais $b=$ cte réalisés sur les 2 appareils. Dans le cas où les lois de comportement utilisées sont écrites en petites déformations et ne prennent en compte ni les rotations matérielles (ou taux de rotation dans les expressions des dérivations de $\sigma$ ) ni l'anisotropie éventuelle du matériau, le résultat des calculs des deux essais pouvaient se déduire l'un de l'autre par simple rotation d'axes.

\subsubsection{Comparaison des résultats}

Les deux sables étudiés sont deux sables fins, Leurs caractéristiques (granulométrie, densité) sont définies en (15).

Les figures 6 et 7 donnent les résultats de cette comparaison pour les deux sables d'Hostun et de Reid Bedfort avec $\sigma_{2}=0,5 \mathrm{MPa}$ et cinq valeurs de $\mathrm{b}$ : $0 ; 0,28 ; 0,5 ; 0,72 ; 1$. Notons que les essais $b=$ 0 et $b=1$ sont axisymétriques et qu'ils sont donc directement comparables $\left(\epsilon_{2}{ }^{\circ}=\epsilon_{2}\right)$.

La figure 6a représente les chemins de contrainte suivis dans le plan déviatoire avec les résistances maximales obtenues. On peut constater qu'elles sont du même ordre sauf pour l'essai $b=0,28$ où la différence importante que l'on observe est probablement due à une localisation prématurée dans l'essai réalisé à la presse 3D.

La figure $6 \mathrm{~b}$ représente l'évolution de la contrainte axiale en fonction de la déformation axiale du cylindre creux et la relation $\sigma_{z}{ }^{*}-\epsilon_{\mathrm{z}}{ }^{*}$ correspondante. Là encore, on obtient une comparaison très acceptable et même parfois inespérée (les courbes $b=1$ et $b=0,66$ sont pratiquement identiques). On remarque également que $\epsilon_{z}{ }^{*}$ change de signe comme $\epsilon_{z}$ entre les deux essais $b=0,5\left(\epsilon_{z}<0\right)$ et $b=0,28$ $\left(\epsilon_{z}>0\right)$. Il existe donc une valeur de $b$ comprise entre 0,5 et 0,28 pour laquelle la déformation axiale du cylindre creux reste nulle.

Pour déterminer plus précisément cette valeur de b (ou $\alpha$ ) nous représentons sur la figure 8 l'orientation $\alpha_{v}$ des lignes d'extension nulle en fonction de b pour les essais à la presse tridimensionnelle. Lorsque la direction axiale du cylindre creux est une direction d'extension nulle on doit également vérifier l'équation $\mathrm{b}=\sin ^{2} \alpha$.

L'intersection des deux courbes donne $b=0,34$ (ou $\alpha=36^{\circ}$ ) qui est bien comprise entre 0,28 et 0,5 .

La figure $6 c$ représente les variations de volume en fonction de $\epsilon_{z}$ pour le cylindre creux et $\epsilon_{\mathrm{z}}{ }^{*}$ pour les essais à la presse 3D. Les résultats ici sont tout à fait remarquables: on observe des serrages initiaux et des pentes de dilatance pratiquement identiques, pour les
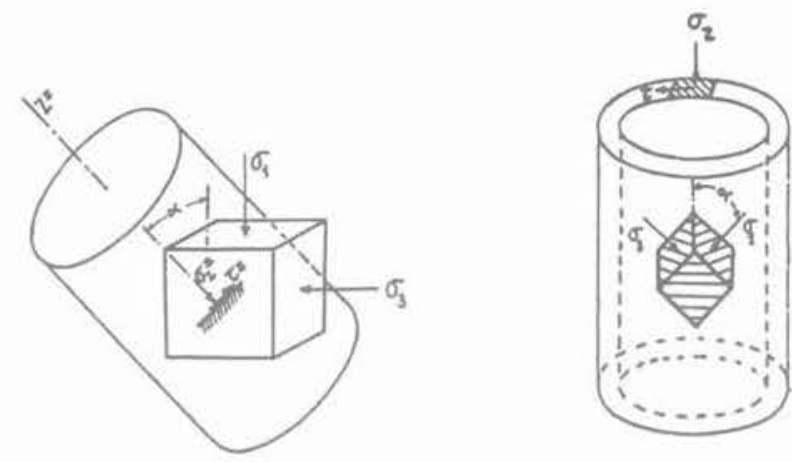

Fig. 5. - Méthode de comparaison des essais sur cylindre creux et presse 3D: définition de $\sigma_{2}^{*}$ et $\tau^{*}\left(b=\sin ^{2} \alpha\right)$.

Fig. 5. - Method of comparison between true triaxial and hollow cylinder data.

deux appareils. Notons que la comparaison pour le sable de Reid Bedford (fig. 7c) est moins bonne.

La figure $6 \mathrm{~d}$ permet de comparer les distorsions $\gamma$ mesurées au cylindre creux et $\gamma^{*}$ calculées. Pour les essais de révolution $b=0$ et 1 les distorsions sont nulles. On a donc tracé seulement les 3 essais correspondants aux valeurs $b=0,28 ; 0,5 ; 0,66$. On peut remarquer ici que systématiquement, pour $\epsilon_{z}$ (ou $\epsilon_{2}{ }^{*}$ ) fixé les distorsions mesurées au cylindre creux sont légèrement supérieures à celles calculées. La comparaison reste cependant très acceptable.

\section{CONCLUSIONS}

La connaissance objective du comportement des matériaux est le but de toute campagne expérimentale, Il est cependant difficile d'estimer l'influence des techniques utilisées par chaque expérimentateur. La comparaison que nous avons menée dans cet article permet d'apporter une réponse partielle à ce problème.

En utilisant les mêmes sables, avec des méthodes de préparations les plus proches possibles, nos deux laboratoires ont réalisés une large campagne d'essais sur deux appareils de conception entièrement différente tant sur la façon d'appliquer le chargement que sur la géométrie de l'échantillon.

Nous avons montré que les résultats obtenus sont souvent très voisins et même qu'ils coïncident parfois de façon inespérée.

La comparaison des courbes de variation de volume et de distorsion en fonction de la déformation axiale nous a montré que les cinématiques suivies sur chaque appareil pour les essais à $b=$ cte sont très proches.

La mobilisation de la résistance (courbes contraintedéformation) est également très comparable surtout pour les essais de compression et d'extension $(b=$ 0 ou 1). Dans les autres cas on observe des diffé- 


\begin{tabular}{|c|c|c|}
\hline$N^{2} d^{\prime}$ Eewal o comparbo & $b$ & Symboleo \\
\hline TRITRI, SFHDS 1 & 0 & $\circ$ \\
\hline Clava., HF1 & 0 & - \\
\hline TRITRI， SFHDS12 & 0.28 & ○ \\
\hline Cleve. HF 14 & 0.28 & - \\
\hline TRITRII SFHDS13 & C. 5 & $\Delta$ \\
\hline Cleve. HF 16 & 0.5 & $\triangle$ \\
\hline TRITRII SFHDS14 & 0.67 & - \\
\hline Cleve.: HF 15 & 0. 72 & - \\
\hline TRITRI: SFHDSIS & 1 & 0 \\
\hline Cleve. HF3 & 1 & 1 \\
\hline
\end{tabular}
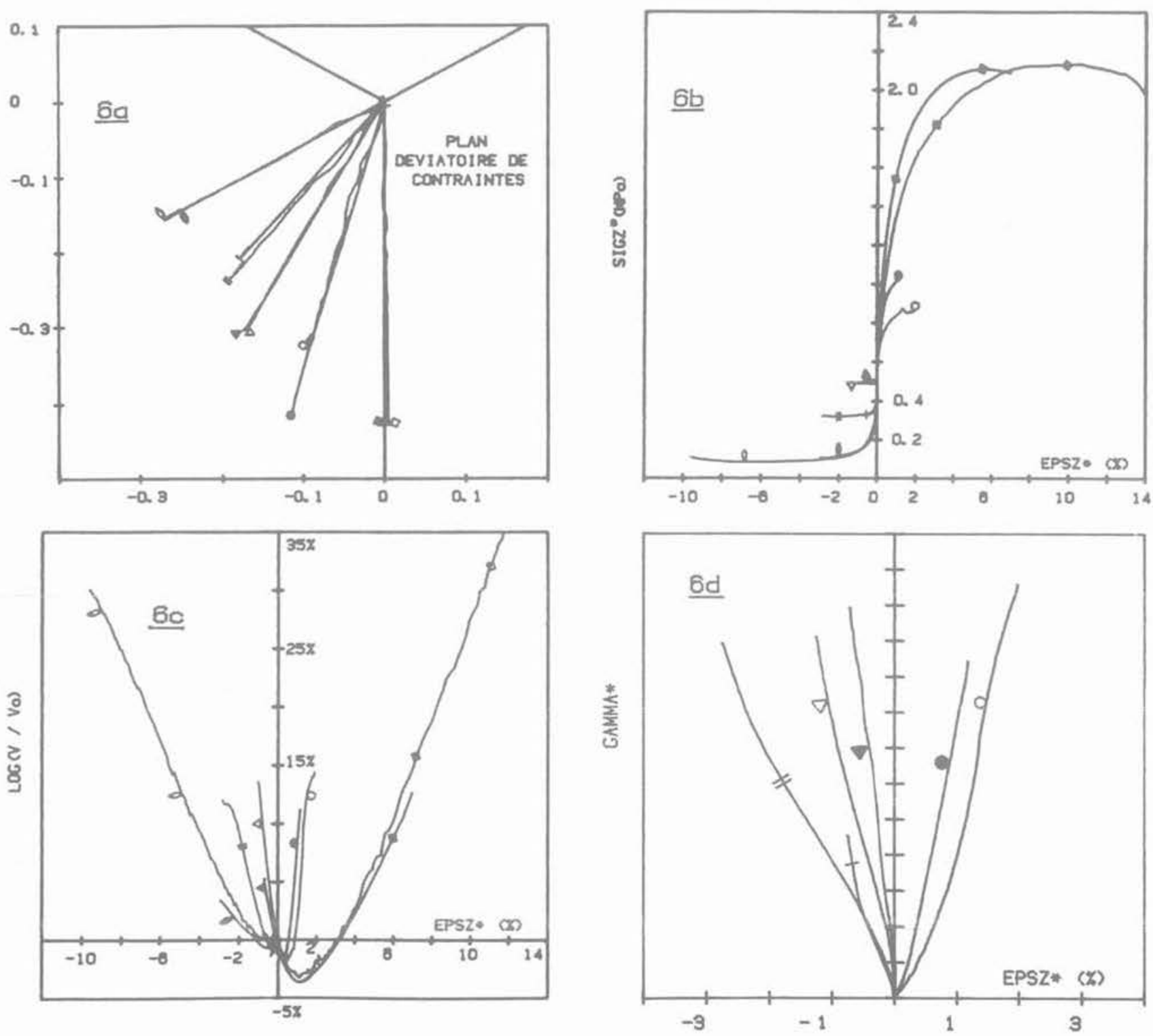

Fig. 6. - Sable d'Hostun: Comparaison des essais $b=c t e$.

Fig. 6. - Comparison of data from radial tests $(b=$ constant $)$ - Hostun sand. 
LECENDE

\begin{tabular}{|c|c|c|}
\hline$N^{\prime} d^{\prime} E \approx a 1 \%$ compará & $b$ & Symboleo \\
\hline TRITRI, RB701 & 0 & $\circ$ \\
\hline Cleva., RA84 & 0 & - \\
\hline TRITRI, RB702 & 0.28 & ○ \\
\hline Cleve.: RAB6 & 0.28 & - \\
\hline TRITRI, RBTO3 & 0.5 & $\Delta$ \\
\hline Cleve. RA9O & 0.5 & $\Delta$ \\
\hline TRITRI: RB704 & 0.67 & $=$ \\
\hline Cleve. RA91 & 0.72 & - \\
\hline TRITRI, RB705 & 1 & 0 \\
\hline Cleve.: RA93 & 1 & 1 \\
\hline
\end{tabular}
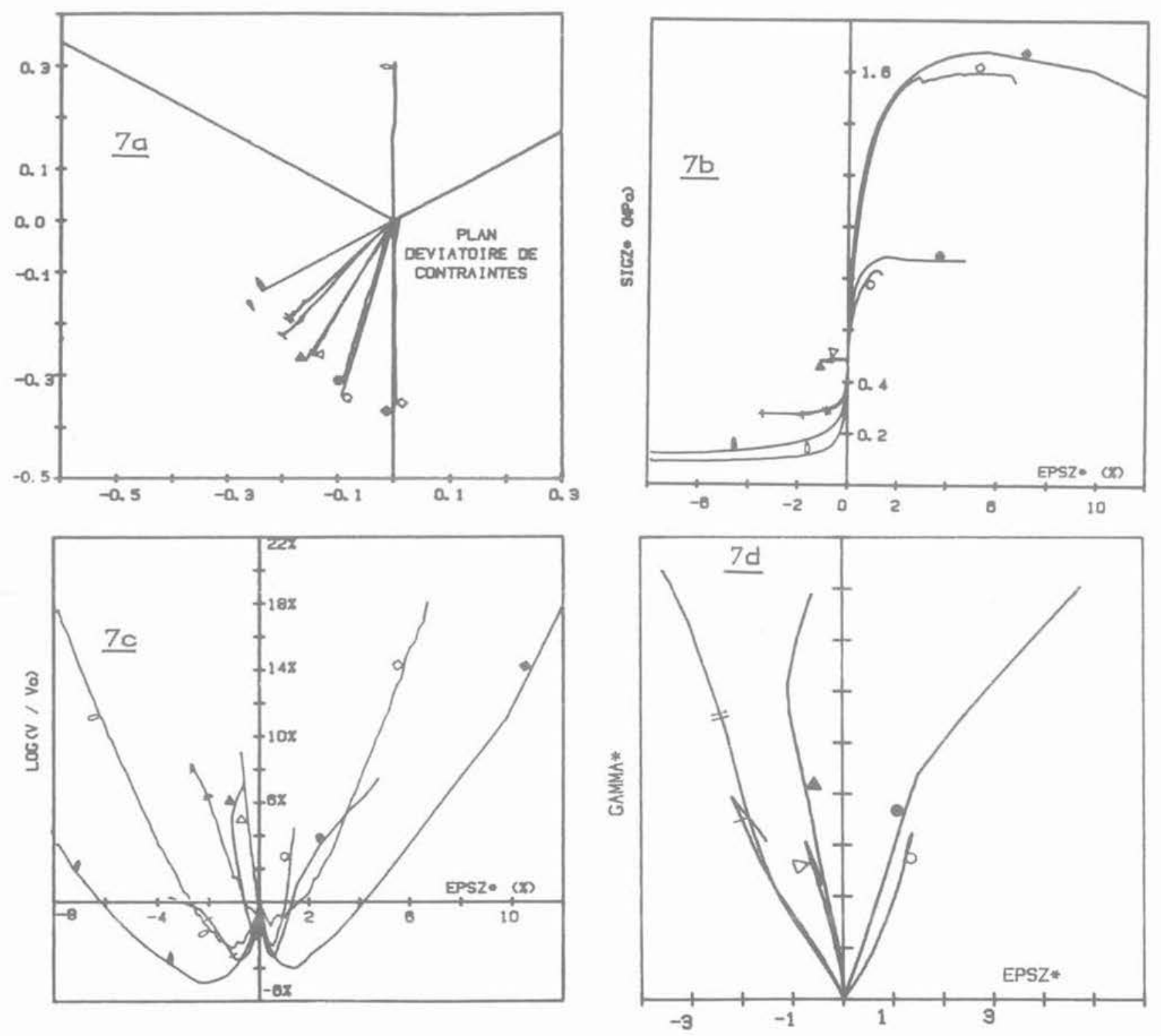

Fig. 7. - Sable de Reid Bedford: Comparaison des essais b = cte.

Fig. 7. - Comparison of data from radial tests $(b=$ constant $)$ - Reid Bedford sand. 

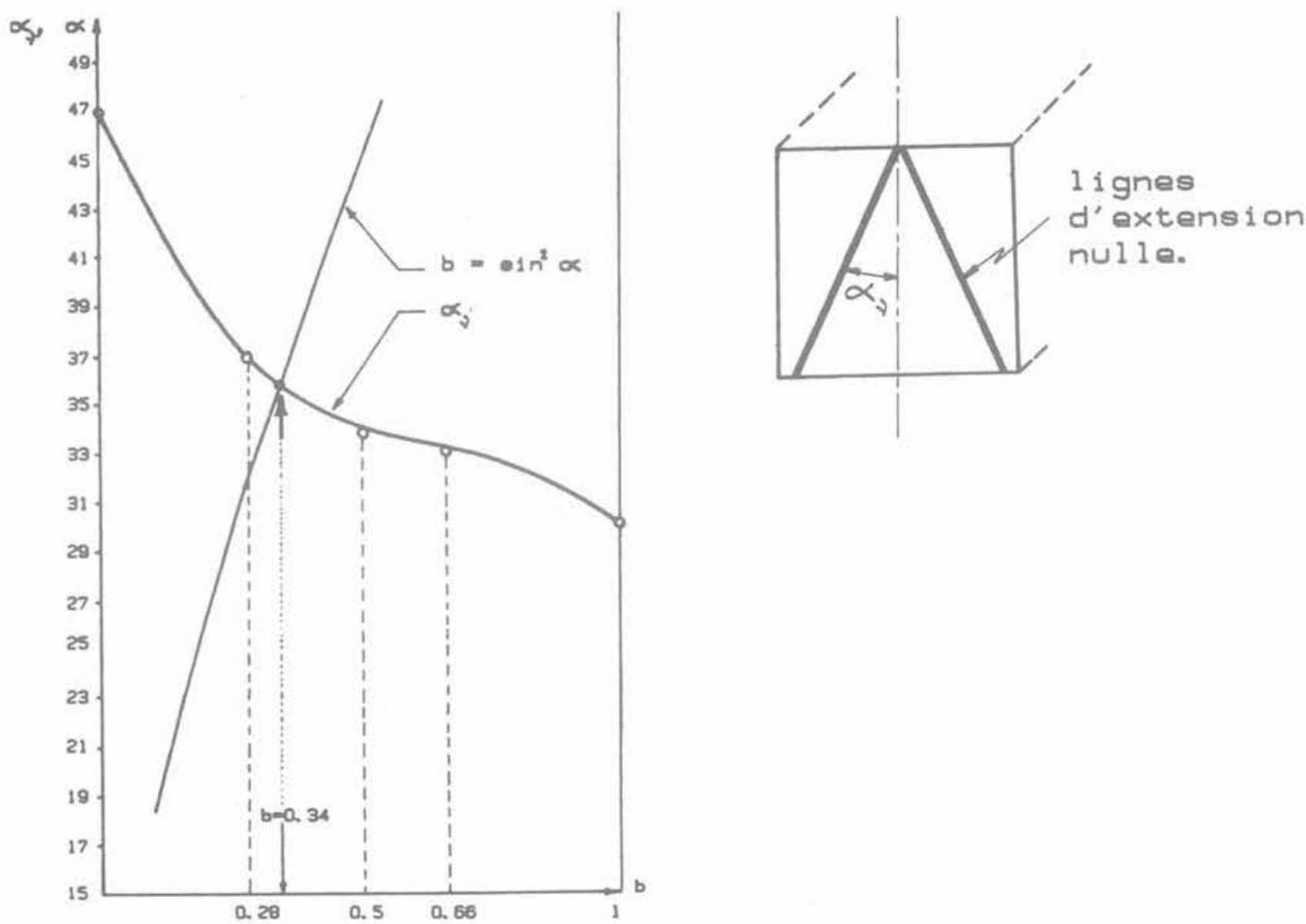

Fig. 8. - Détermination de la valeur de b correspondant à une déformation axiale nulle au cylindre creux. Fig. 8. - Graphical determination, from true triaxial data, of the b-value corresponding to no-extension of the axial direction in hollow cylinder.

rences variables sur les modules initiaux et les niveaux de résistance maximale.

Dans tous les cas, les différences observées entre les résultats du cylindre creux et de la presse 3D peuvent être considérés comme étant du même ordre de grandeur que la dispersion que lon obtient sur la presse 3D lorsque l'on répète le même essai (en particulier les essais non axisymétriques).

En conclusion, les deux appareils permettent d'approcher de façon qualitative et quantitative le comportement rhéologique tridimensionnel du matériau testé.

La campagne que nous avons menée permet également de conclure que l'anisotropie initiale des échantillons est faible puisque les mêmes réponses sont obtenues pour 2 orientations différentes de l'état de contrainte. En particulier les différentes valeurs de la résistance maximale, sont dues essentiellement aux différentes valeurs du paramètre $b$ et non de $\alpha$.

Une difficulté subsiste cependant pour apprécier le comportement en compression isotrope: d'une part des corrections dues aux effets de membrane semblent nécessaires mais ces corrections se révèlent être du même ordre de grandeur que les quantités mesurées. D'autre part, ces essais où les déformations sont faibles sont très sensibles au mode de préparation. Nous avions déjà rencontré le même problème en comparant des essais réalisés sur les deux presses tridimensionnelles de Karlsruhe et Grenoble (18).

\section{BIBLIOGRAPHIE}

1. SAADA A.S., TOWNSEND F.C. (1981), Laboratory strength testing of soil (State of Art), in Laboratory shear strength of soil, ASTM STP 740 ASTM, Philadelphia, pp. 7-77.

2. LANIER J. (1987), Développements récents des essais en laboratoire. Manuel de Rhéologie des Géomatériaux, F. Darve éd., Presses des Ponts et Chaussées, Paris, pp. 15-31.

3. SAADA A.S. (1988), Hollow cylinder torsional devices: their advantages and limitations. Advanced triaxial testing of soil and rock ASTM STP 977. R.T. Donaghe, R.C. Chaney, M.L. Silver, Edits, ASTM Philadelphia, pp. 766-795.

4. Workshop on limit equilibrium, plasticity and generalized stress-strain relations in geotechnical engineering, Mc Gill University, Montréal, 1980. 
5. Constitutive relations for soils. Proc. Workshop, Grenoble, Gudehus G., Darve F., Vardoulakis 1. eds, Balkema, Rotterdam, 1982.

6. Constitutive equations for granular non-cohesive soils, Proc. Workshop Cleveland, A. Saada, G. Bianchini eds, Balkema, Rotterdam, 1989.

7. FLAVIGNY E., LEFEBVRE P. (1987), Etucie de la répétabilité des essais triaxiaux de révolution. Rapport scientifique GRECO Géomatériaux, Institut de Mécanique de Grenoble, pp. 200-204.

8. Banque de données MODELISOL, J.L. Favre, P.Y. Hicher, Ecole Centrale de Paris, Chatenay Malabry.

9. LANIER J. (1988), Special stress paths along the limit surface of a sand specimen with the use of a true Triaxial apparatus. Advanced triaxial testing of soil and rock, ASTM STP 977 , pp. 859-869.

10. ZITOURNI Z. (1988), Comportement tridimensionnel des sables. Thèse, Grenoble.

11. LANIER J., ZITOUNI Z. (1989), Development of a data base using the Grenoble true triaxial apparatus, Constitutive equations for granular non-cohesive soils. A. Saada, G. Bianchini ed., Balkema, pp. 47-58.

12. HIGHT D.W., GENS A., SYMES M.J. (1983), The development of a new hollow cylinder appa- ratus for investigating the effect of principal stress rotation. Géotechnique, vol. 33, pp. 355-383.

13. KARCHAFI M. (1988), Contribution à létude du comportement des matériaux granulaires sous sol. licitations rotationnelles. Thèse, Ecole Centrale de Paris.

14. KARCHAFI M. (1989), On effects of sample height in hollow cylinder tests, Constitutive equa. tions for granular non-cohesive soils. A. Saada, G. Bianchini ed., Balkema, pp. 41-45.

15. SAADA A., PUCCINI P. (1989), The develop. ment of a data base using the Case hollow cylin. der apparatus, Constitutive equations for granu. lar non-cohesive soils. A. Saada, G. Bianchini ed., Balkema, pp. 33-40.

16. SAADA A., BIANCHINI G., PUCCINI P. (1983), The mechanical properties of anisotropic granular soils. Colloque international du CNRS, Grenoble.

17. CAMBOU B., DOGUI A., JAFARI K., SIDOROFF F. (1986), On the equivalence between true triaxial and torsion shear loading paths. Computers and Geotechnics 2, pp. 207-217.

18. LANIER J., STUTZ P. (1982), Supplementary true triaxial tests in Grenoble. Constitutive Relations for Soils, Gudehus, Darve, Vardoulakis eds, Balkema, Rotterdam, pp. 67-70. 